\title{
Effects of Link Capacity Reductions on the Reliability of an Urban Rail Transit Network
}

\author{
Jie Liu $\mathbb{D}^{1},{ }^{1,2,3}$ Paul M. Schonfeld, ${ }^{4}$ Yong Yin $\mathbb{D}^{1,2,3}$ and Qiyuan Peng $\mathbb{D}^{1,2,3}$ \\ ${ }^{1}$ School of Transportation and Logistics, Southwest Jiaotong University, Chengdu 611756, China \\ ${ }^{2}$ National United Engineering Laboratory of Integrated and Intelligent Transportation, Southwest Jiaotong University, \\ Chengdu 611756, China \\ ${ }^{3}$ National Engineering Laboratory of Integrated Transportation Big Data Application Technology, Southwest Jiaotong University, \\ Chengdu 611756, China \\ ${ }^{4}$ Department of Civil and Environmental Engineering, University of Maryland, College Park 20742, MD, USA
}

Correspondence should be addressed to Yong Yin; yinyong@swjtu.edu.cn

Received 7 February 2020; Revised 11 September 2020; Accepted 5 October 2020; Published 29 October 2020

Academic Editor: Prakash Ranjitkar

Copyright (C) 2020 Jie Liu et al. This is an open access article distributed under the Creative Commons Attribution License, which permits unrestricted use, distribution, and reproduction in any medium, provided the original work is properly cited.

Link capacity reductions, which occur often, degrade the service quality and performance of urban rail transit (URT) networks. To measure the reliability of a URT network when link capacity reductions occur in a given time period, the passengers' generalized travel cost (GTC) is computed and passengers are divided into three categories. The GTC considers here the crowding in trains, seat availability, and perceived travel time. Passengers whose relative increase in GTC on a URT is below or above a preset threshold belong to category I or II, respectively, while passengers who cannot travel on the URT due to insufficient capacities on their paths belong to category III. Passenger trips in categories I are acceptable since their GTC increases only slightly with link capacity reductions. The fraction of acceptable trip (FAT) and total GTC increase ratio (TGCR) in a given time period are defined here as the network's reliability and unreliability metrics, respectively. The ratio of affected passenger trip (RAPT) is proposed to identify each line's most critical links. The reliability and unreliability metrics of Wuhan's URT network during evening peak hours are computed when the capacities of the most critical link or multiple most critical links are reduced. The results show that the proposed RAPT indicator is effective in identifying the most critical links that greatly affect the reliability and performance of a URT network. For capacity reductions on a line's most critical link, the proposed method can determine the capacity reduction ratio corresponding to network's high FAT and low TGCR as well as the priorities of lines needing emergency measures to maintain high network reliability and performance. For capacity reductions on critical links of multiple lines, the proposed method can identify the number of reduction links and the capacity reduction ratio that the network can withstand while maintaining its reliability and performance above a specified level.

\section{Introduction}

To deal with the urban challenges of congestion, noise, and emissions due to rising demand, urban rail transit (URT) development is promoted by China's government for its desirable economic, social, and environmental benefits [1]. Hence, URT networks in large urban areas have expanded rapidly in recent years and have a major role in public transport. As economic and social development advances, passengers expect from high quality and reliable transportation services from URT [2, 3]. However, URT networks are often subject to disturbances (e.g., public events, vehicle breakdowns, and equipment failures) in daily operations. Those disturbances cause link capacity reductions on the URT network, which results in degraded network performance and low reliability. As passenger volume on a URT network increases, the sensitivity of the network to the disturbances also increases. This may inhibit the passengers' willingness to shift from private transportation to public transportation. Accordingly, measuring and increasing the reliability of a URT network when link capacity reductions 
occur are essential in URT policymaking, planning, and operations.

The rest of the paper is organized as follows: first, the related work is presented. Second, the metrics for measuring the reliability and unreliability of a URT network are proposed. A traffic assignment model is introduced to compute the metrics when link capacity reductions occur. Third, the reliability and unreliability of Wuhan's URT network during evening peak hours when link capacity reductions occur is analyzed. Finally, the main conclusions are summarized and suggestions are offered for maintaining the high reliability and performance of a URT network.

1.1. Literature Review. To measure the probability of disturbances and the impacts of disturbances on transportation networks as well as networks' ability to absorb disturbances, many studies use concepts such as reliability, vulnerability, robustness, and resilience [4]. Vulnerability is defined as the susceptibility to disturbances, which emphasizes measurement of the consequences of disturbances [5]. Robustness represents the ability to maintain system performance under disturbances, which is the inverse concept of vulnerability [6]. The resilience of a transportation network is defined as the network's ability to resist, absorb, adapt to, and recover from negative impacts of disturbances [6]. The reliability of a transportation network has been defined in dissimilar ways in various studies. Bell defines that a network is reliable if the expected trip costs are acceptable even when passengers are very pessimistic about the state of the network [7]. Kim et al. define the reliability of a transportation network as the possibility that the network performed its intended tasks satisfactorily for a certain period of time $[8,9]$. Gu et al. specify that transportation network reliability is the probability that the network could remain satisfactory in terms of service level provision when perturbation occurs [4].

In practice, the degradation of a transport network could be due to many interruptions, such as operational accidents, natural disasters, and terrorist activities [10]. Those serious events cause complete failures of stations or links. The vulnerability of the transport network was measured based on network performance with and without complete failures at stations or links. Many network performance metrics were proposed that reflected the topology of the transport network [11], such as global connectivity (the minimum number of links whose removal disconnects the remaining nodes from each other), the largest connected component of the network, average shortest-path length, and other derivative metrics [12-15]. The vulnerability and resilience of a URT network were measured according to the relative differences in the above metrics due to network damage events compared to the normal situation. Simulations that removed stations or links were performed to analyze the vulnerability, robustness, and reliability of URT networks $[12-14,16]$. The influence of removing stations and links on topologies of URT networks was analyzed deeply in the above studies. The research focused on connectivity issues when the network becomes disconnected due to complete failures at stations or links
[17-19]. Although the network performance and service quality of URT's were heavily affected by total loss of station or link capacities, the likelihood of such serious events is low. On the contrary, small disturbances that reduce the capacities of links and stations often occur. In addition, the passengers' travel behavior and transport capacity were neglected in some studies when measuring the vulnerability, reliability, resilience, and robustness of URT networks. For example, Sun et al. analyzed the impact of removing stations on passengers' volume but without considering passengers' travel behaviors and the transportation service quality [20]. Kim and Song measured the reliability of a mass transit system based on the topological structure of the network and the passenger flow among pairs of stations [21]. They also neglected the effects of passengers' travel path selection behaviors on the reliability of a transportation network. Lu measured the resilience of the Shanghai metro network by integrating the network topology and passenger volume [22]. He assumed that passengers travel on the shortest travel paths between pairs of stations. De-Los-Santos et al. measured the robustness of a rail transit network when links were removed from it based on the passengers' overall travel time [23]. However, they assumed that passengers only chose the shortest paths and waited for the failure to be repaired when no alternative path existed, which was inconsistent with passengers' travel characteristics.

Researchers had noted the necessity of considering traffic demand and transport supply in studying the reliability, vulnerability, and robustness of transport networks $[6,24]$. Snelder et al. also proposed a framework for analyzing the robustness of road networks for short-term variations in supply [25]. Considering traffic demand and transportation supply could measure the influence of full and partial link capacity reductions on network performance, which helped to measure the reliability, vulnerability, and robustness of transportation networks. Jiang et al. assessed the vulnerability of the transport network that considers both equilibrium flows and the probability of the disturbance [26]. Cats and Jenelius researched the impact of partial capacities degradation of lines on public transport network vulnerability [27]. Jenelius measured the reliability of a high-frequency bus line in Stockholm in terms of the passengers' perceived travel time [28]. While he analyzed the service reliability when bus lines operate normally, the effects of transport interference on the reliability of bus lines had not been analyzed. Liu et al. measured the travel reliability of a URT network with connectivity reliability, travel time reliability, and capacity reliability during normal operations of the URT network [24]; although passengers' route choices behavior was considered, the travel reliability of a URT network under disturbances was not measured.

The transportation network reliability is closely related to the acceptable level of service. Regarding the reliability of public transportation network reliability, many researchers had defined the acceptable level of service in terms of punctuality (i.e., on-time performance) and the coefficient of variation of vehicle headways. However, most standards for the acceptable level of service are proposed from the 
perspective of suppliers rather than passengers $[29,30]$. In addition, the standards focused on travel time or vehicle running time, which reflects the extent to which vehicles ran on time. However, the transportation service quality perceived by passengers on the journey is neglected. Even if passengers arrive at their destination on time, they may consider their travel unacceptable because of low perceived service quality to passengers.

1.2. Objective and Contribution. This paper evaluates the reliability and unreliability of a URT network when link capacity reductions occur. It analyzes cases not only where the link capacity is reduced to zero, but also cases where the link capacity is partially reduced. The passengers' generalized travel cost (GTC) which is the sum of the monetary value of perceived travel time and monetary cost of the trip is computed to reflect the service quality perceived by passengers. If link capacity reductions occur, the reliability of the URT network decreases because some passengers' perceived service quality is not acceptable. To determine whether the service perceived by a passenger is acceptable, the relative increase in GTC for passengers is computed. Passengers are classified into three categories according to their relative increase in GTC and whether paths with spare capacity exist between station pairs when link capacity reductions occur. Passengers whose relative increase in GTC on a URT is below or above a preset threshold belong to category I or II, respectively. With link capacity reductions, passengers in categories I and II can travel on a URT network. Passengers who cannot travel on the URT due to insufficient capacities on their paths belong to category III. The fraction of acceptable trips (FAT) is proposed to measure the network reliability within a given time period. Only passengers in category I perceive acceptable service when link capacity reductions occur, since their GTC increases only slightly. Thus, the FAT equals the fraction of passengers in category I, which is defined as the metric of URT network reliability within a given time period.

Passengers' total GTC is used to reflect the network performance of a URT. The difference in passengers' total GTC with and without link capacity reductions measures the impact of link capacity reductions on the URT network. The total GTC increase ratio (TGCR) is defined as the metric of the unreliability of a URT network when link capacity reductions occur.

To identify lines' most critical links, the ratio of affected passenger volume (RAPT) is proposed. Finally, the proposed indicators and method are applied to Wuhan's URT network during evening peak hours. The reliability of Wuhan's URT network during evening peak hours is computed when the capacities of each line's most critical link or multiple lines' most critical links are reduced. The proposed indicators and method help to identify each line's most critical links, as well as the extent and the relations among the capacity reductions on lines' most critical link and network performance, thereby supporting infrastructure management and capacity allocation.

\section{Model Formulation}

2.1. URT Network and Passengers' Categories. A weighted and directed graph $G=(S, E)$ is used to represent a URT network, which consists of the set of URT stations $S$ and the set of URT links $E \subseteq\{(i, j) \mid i, j \in S, i \neq j\}$ that connect stations directly.

Passengers in categories I and II can travel on the URT network. However, only passengers in category I get acceptable service and their trips are acceptable when link capacity reductions occur, since their GTC increases only slightly. $\left(E_{\text {redu }}, x\right)$ represents that the capacities of link set $E_{\text {redu }}, E_{\text {redu }} \in E$ are reduced by reduction ratio $x, x \in(0,100 \%]$. If $x=100 \%$, then capacities of links in the set $E_{\text {redu }}$ are reduced to zero. A passenger $n$ traveling from station $i$ to station $j$ is represented as $p_{\mathrm{ij}}^{n}$. The category of the passenger $p_{\mathrm{ij}}^{n}$ is determined by equations (1) and (2) when link capacity reductions occur:

$$
\begin{gathered}
\lambda=\frac{C_{\mathrm{ij}}^{n}\left(E_{\text {redu }}, x\right)-C_{\mathrm{ij}}^{n}(0,0)}{C_{\mathrm{ij}}^{n}(0,0)}, \\
p_{\mathrm{ij}}^{n} \in \begin{cases}P_{1}^{\mathrm{ij}}\left(E_{\mathrm{redu}}, x\right) ; & \lambda \leq \kappa \cap\left|p a_{\mathrm{ij}}\right|>0 ; \\
P_{2}^{\mathrm{ij}}\left(E_{\text {redu }}, x\right) ; & \kappa>\lambda \cap\left|p a_{\mathrm{ij}}\right|>0 ; \\
P_{3}^{\mathrm{ij}}\left(E_{\text {redu }}, x\right) ; & \left|p a_{\mathrm{ij}}\right|=0 ;\end{cases}
\end{gathered}
$$

where $\lambda$ is the relative increase in GTC for passenger $p_{\mathrm{ij}}^{n}$ when link capacity reductions occur, $C_{\mathrm{ij}}^{n}(0,0)$ and $C_{\mathrm{ij}}^{n}\left(E_{\text {redu }}, x\right)$ are the GTCs of passengers $p_{\mathrm{ij}}^{n}$ from station $i$ to station $j$ when a URT network operates normally and when capacities of link set $E_{\text {redu }}$ are reduced by $x$, respectively, $P_{1}^{\mathrm{ij}}\left(E_{\mathrm{redu}}, x\right), P_{2}^{\mathrm{ij}}\left(E_{\text {redu }}, x\right)$, and $P_{3}^{\mathrm{ij}}\left(E_{\mathrm{redu}}, x\right)$ are the sets of passengers from station $i$ to station $j$ and belonging to categories I, II, and III, respectively, when capacities of link set $E_{\text {redu }}$ are reduced by $x,\left|\mathrm{pa}_{\mathrm{ij}}\right|$ is the number of paths with spare capacity from station $i$ to station $j$, and $\kappa$ is a preset threshold.

2.2. GTC Computation. The passengers' GTC is the sum of the monetary and nonmonetary costs of a trip [31-33]. Nonmonetary cost refers to the perceived travel time undertaking the journey. The perceived travel time is converted to a monetary value using a value of time, which usually varies according to the traveler's income, trip component, and trip purpose. The travel time from a station to another is separated into different components, i.e., walking time, waiting time, and in-vehicle time. Research shows that the different components of travel time are perceived by passengers differently, e.g., waiting time has a much higher perceived value than in-vehicle travel time when the vehicle is not crowded. In addition, seat availability and crowding in the vehicle also affect passengers' perceived in-vehicle time. Therefore, each part of the travel time has its own value and weighting [34]. When a URT network operates normally, passenger $p_{\mathrm{ij}}^{n}$ s $\mathrm{GTC}$ from station $i$ to station $j$ is computed with the following equation: 


$$
C_{\mathrm{ij}}^{n}(0,0)=t_{\mathrm{ij}}^{n}(0,0) \cdot \alpha+c_{\mathrm{ij}},
$$

where $C_{\mathrm{ij}}^{n}(0,0)$ is the passenger $p_{\mathrm{ij}}^{n}$ 's GTC from station $i$ to station $j$ when a URT network operates normally, $t_{\mathrm{ij}}^{n}(0,0)$ is the passenger $p_{\mathrm{ij}}^{n}$ 's perceived travel time from station $i$ to station $j$ when a URT network operates normally, $c_{i j}$ is fare from station $i$ to station $j$, which is not affected by link capacity reductions, and $\alpha$ is the value of time figure that converts the perceived travel time into money.

The perceived travel time includes perceived waiting time (waiting time at origin and transfer stations), perceived walking time (access, egress, and transfer walking time), perceived in-vehicle time considering seat availability and crowding in the vehicle, and increased perceived travel time due to transfer $[27,35]$. Each perceived travel time component equals time multiplied by time weights. Passenger $p_{\mathrm{ij}}^{n}$ 's perceived travel time from station $i$ to station $j$ when a URT network operates normally is computed with the following equation:

$$
\begin{aligned}
t_{\mathrm{ij}}^{n}(0,0) & =\beta^{\text {wait }} \cdot t^{\text {wait }}(0,0)+\beta^{\text {walk }} \cdot t^{\text {walk }}(0,0) \\
& +\beta^{\text {crow }} \cdot t^{\text {inv }}(0,0)+\beta^{\text {trans }} \cdot n^{\text {trans }}(0,0),
\end{aligned}
$$

where $t^{\text {wait }}(0,0), t^{\text {inv }}(0,0), t^{\text {walk }}(0,0)$, and $n^{\text {trans }}(0,0)$ are the waiting time, walking time, in-vehicle time, and transfer times, respectively, when a URT network operates normally. The $\beta$ values are the corresponding time weights.

Passengers' waiting time, transfer times, and crowding in vehicles increase while seat availability decreases when link capacity reductions occur. Therefore, passengers' GTC increases when the capacities of link set $E_{\text {redu }}$ are reduced by $x$. If passenger $p_{\mathrm{ij}}^{n}$ belongs to categories I and II, then his GTC $C_{\mathrm{ij}}^{n}\left(E_{\mathrm{redu}}, x\right)$ can be computed with equations (3) and (4) when capacities of link set $E_{\text {redu }}$ are reduced by $x$. However, if passenger $p_{\mathrm{ij}}^{n}$ belongs to category III, then his GTC is assumed to be the maximum GTC of passengers in categories I and II when the URT network operates normally.

2.3. Metrics of the Reliability and Unreliability of a URT Network. Passengers in categories I and II still can travel on the URT network when link capacity reductions occur. Nevertheless, passengers in category I are only slightly affected by link capacity reductions and their perceived service is acceptable. Thus, the FAT is the fraction of passengers in category I which is defined as the metric of the reliability of a URT network in a given time period, which is computed with the following equation:

$$
R_{\mathrm{re}}\left(E_{\mathrm{redu}}, x\right)=\frac{\sum_{i \in S} \sum_{j \in S, j \neq i}\left|P_{1}^{\mathrm{ij}}\left(E_{\mathrm{redu}}, x\right)\right|}{\sum_{i \in S} \sum_{j \in S, j \neq i} v_{\mathrm{ij}}}
$$

where $R_{\mathrm{re}}\left(E_{\mathrm{redu}}, x\right)$ is the metric of the reliability of a URT network when capacities of link set $E_{\text {redu }}$ are reduced by $x$, $\left|P_{1}^{\mathrm{ij}}\left(E_{\mathrm{redu}}, x\right)\right|$ is the number of passengers trips who travel from station $i$ to station $j$ and belong to category I in a given time period, and $v_{\mathrm{ij}}$ is passenger trips traveling from station $i$ to station $j$ in a given time period.

The total GTC can be used to evaluate the performance of the URT network [27]. The reliability of a URT network cannot be measured comprehensively by only using as a metric of the fraction of passengers in category I (e.g., Case 1: the fractions of passengers in categories I, II, and III are 0.8, 0.1 , and 0.1, respectively; Case 2: the fractions of passengers in categories I, II, and III are $0.8,0.15$, and 0.05 , respectively.) The reliability in Cases 1 and 2 is the same. However, the network performance in Case 2 is better than Case 1, since passenger' GTC in category III is higher than passengers' GTC in category II. Therefore, the effects of link capacity reductions on the URT network are measured as the difference in passengers' total GTC with and without link capacity reductions. Passengers' total GTC in a given time period is computed with equation (6) when a URT network operates normally.

$$
C(0,0)=\sum_{i \in S} \sum_{j \in S, j \neq i} \sum_{n=1}^{v_{\mathrm{ij}}} C_{\mathrm{ij}}^{n}(0,0)
$$

where $C(0,0)$ is the passengers' total GTC when the URT network operates normally.

Equation (6) can be applied to computed passengers' total GTC $C\left(E_{\text {redu }}, x\right)$ when capacities of link set $E_{\text {redu }}$ are reduced by $x$. The TGCR is defined as the metric of the unreliability of a URT network when capacities of link set $E_{\text {redu }}$ are reduced by $x$, which is computed with the following equation:

$$
R_{\mathrm{un}}\left(E_{\mathrm{redu}}, x\right)=\frac{C\left(E_{\mathrm{redu}}, x\right)-C(0,0)}{C(0,0)}
$$

where $R_{\mathrm{un}}\left(E_{\mathrm{redu}}, x\right)$ is the metric of the unreliability of a URT network when capacities of link set $E_{\text {redu }}$ are reduced by $x$.

2.4. Simulation Based on Traffic Assignment. In order to measure the reliability of a URT network when link capacity reductions occur, a logit-based stochastic user equilibrium model, which has the advantage of reflecting passengers' travel behavior and the congestion effect $[36,37]$, is used to compute passengers' GTC and determine passengers' categories. The model is formulated as equations (8)-(16) and solved by the successive weighted averages method [38]:

$$
\min Z(f)=-\sum_{i \in S} \sum_{j \in S} \sum_{m \in M_{\mathrm{ij}}} v_{\mathrm{ij}}^{m} \cdot E\left(C_{\mathrm{ij}}, \theta\right)+\sum_{e \in E} \mathrm{fl}_{e} \cdot C_{e}\left(\mathrm{fl}_{e}\right)-\sum_{e \in E} \int_{0}^{\mathrm{fl}_{e}} C_{e}(\omega) \mathrm{d} \omega
$$


subject to

$$
\begin{aligned}
& \sum_{m} v_{\mathrm{ij}}^{m}=v_{\mathrm{ij}}, \\
& v_{\mathrm{ij}}^{m}=\frac{\exp \left(-\theta \cdot C_{\mathrm{ij}}^{m}\right)}{\sum_{m \in M_{\mathrm{ij}}} \exp \left(-\theta \cdot C_{\mathrm{ij}}^{m}\right)} \cdot v_{\mathrm{ij}}, \\
& E\left(C_{\mathrm{ij}}, \theta\right)=-\frac{1}{\theta} \operatorname{In} \sum_{m \in M_{\mathrm{ij}}} \exp \left(-\theta \cdot C_{\mathrm{ij}}^{m}\right), \\
& \mathrm{fl}_{e}=\sum_{i} \sum_{j} \sum_{m \in M_{\mathrm{ij}}} v_{\mathrm{ij}}^{m} \cdot \delta_{e, m}^{\mathrm{ij}}, \\
& \mathrm{Cap}_{e}=f_{e} \cdot n_{c}, \\
& \mathrm{fl}_{e} \leq \mathrm{Cap}_{e}, \\
& \psi_{e}=n_{s} \cdot f_{e}, \\
& C_{e}(\omega)=\beta^{\mathrm{crow}} \cdot t_{e}^{\mathrm{inv}},
\end{aligned}
$$

where $\theta$ is a nonnegative parameter that represents the accuracy of passengers' perception of travel cost, $v_{\mathrm{ij}}^{m}$ is the volume of passenger trips from station $i$ to station $j$ on path $m$, which is computed with the logit model of equation (10), $C_{\mathrm{ij}}^{m}$ is the GTC of path $m$ from station $i$ to station $j, v_{\mathrm{ij}}$ is the passenger trip volume from station $i$ to station $j, E\left(C_{\mathrm{ij}}, \theta\right)$ is the mathematical expectation of passengers for GTC perception, $C_{\mathrm{ij}}=\left\{\cdots, C_{\mathrm{ij}}^{m}, \cdots\right\}$ is the GTC set corresponding to paths from station $i$ to station $j, \mathrm{fl}_{e}$ is the passenger flow on link $e$, which is the sum of passenger flows on paths that contain link $e$, and $\delta_{e, m}^{\mathrm{ij}}$ is a $0-1$ binary variable. If the path $m$ from station $i$ to station $j$ contains the link $e$, then $\delta_{e, m}^{1 j}=1$; otherwise, $\delta_{e, m}^{\mathrm{ij}}=0$. Cap $\mathrm{Ca}_{e}$ is the capacity of the link $e$, which is the number of trains $f_{e}$ traversing link $e$ per hour multiplied by the maximum allowable number of passengers per train $n_{c} . \psi_{e}$ is the number of seats on link $e$, which equals the seats of per train $n_{s}$ multiplied by the number of trains running $f_{e}$ on the link $e$ per hour. $C_{e}(\omega)$ is the functional relation between travel cost in a link and the link's passenger flow $\omega$, which is expressed in equation (16). $t_{e}^{\text {inv }}$ is the in-vehicle time on link $e$.

2.5. Identification of Critical Links. The betweenness of links has been widely used to identify the critical links on a URT network [23]. The betweenness of a URT link is the ratio of the number of the shortest paths containing that link to the number of all shortest paths between OD pairs. It is computed with the following equation:

$$
B_{e}=\sum_{i \in N} \sum_{j \in N, j \neq i} \frac{n_{\mathrm{ij}}^{e}}{n_{\mathrm{ij}}}, \quad \forall e \in E,
$$

where $B_{e}$ is the betweenness of link $e, n_{\mathrm{ij}}$ is the number of shortest paths from station $i$ to station $j$, and $n_{\mathrm{ij}}^{e}$ is the number of shortest paths from station $i$ to station $j$ which contains link $e$.

The betweenness of links is proposed from the topology of a URT network. It measures the criticality of links from the shortest paths on a URT network. However, it cannot measure the effects of link disruptions on passengers' travel comprehensively, since it neglects the passenger flow on the URT network. The ratio of affected passenger trips (RAPT) is proposed to measure the passenger trips that a link can affect, i.e., the passenger trips whose travel paths include that link. It is used to identify critical links and specified with the following equation:

$$
I_{e}=\frac{\sum_{i \in S} \sum_{j \in S, j \neq i} \sum_{m \in M_{\mathrm{ij}}} v_{\mathrm{ij}}^{m} \cdot \delta_{e, m}^{\mathrm{ij}}}{\sum_{i \in S} \sum_{j \in S, j \neq i} v_{\mathrm{ij}}}, \quad \forall e \in E,
$$

where $I_{e}$ is the ratio of affected passenger trips who is affected by link $e$, which is computed based on a logit-based stochastic user equilibrium model.

\section{Application}

3.1. Case Study Description. Wuhan's URT network is used in a case study to measure its reliability when link capacity reductions occur. Wuhan's URT network connects three parts of Wuhan (Wuchang, Hankou, and Hanyang) which are divided by Yangtze and Han rivers (the black dotted lines in Figure 1). Wuhan's URT included 7 lines and 149 stations (17 transfer stations) in September 2018. Figure 1 shows the operating lines, station numbers, and abbreviations of station names for Wuhan's URT in September 2018.

3.2. Data Preparation. Automatic Fare Collection (AFC) data records for 5 working days in September 2018 are obtained from Wuhan's URT operator to measure the reliability of that URT network during evening peak hours (5: 30-7:30). The average OD (origin station to destination station) trip distribution is computed using AFC data. The waiting time at origin station and transfer station is estimated as half of the lines' headway (Dixit et al. 2019 and DeLos-Santos et al. 2012) [23, 39]. The access walking time and egress walking time at different stations are different. Here, we assume that access walking time or egress walking time at a station is 4 minutes, according to our survey. The hourly capacities of lines and the number of seats per hour on lines (links have the same capacity and same seats along a line), the headway of lines, the in-vehicle travel time (including dwell time and train running time) of links, and the transfer walking time at transfer stations are obtained from an operator. The capacities, headway, and number of seats of lines during evening peak hours are shown in Table 1. Taking line 1 as an example, the transfer walking time at transfer stations and in-vehicle travel time of some links on line 1 are shown in Tables 2 and 3, respectively, during evening peak hours.

The preset threshold which is used to determine passengers' categories when link capacity reductions occur is obtained by conducting a questionnaire survey of passengers in Wuhan's URT network. A total of 1427 questionnaires are 


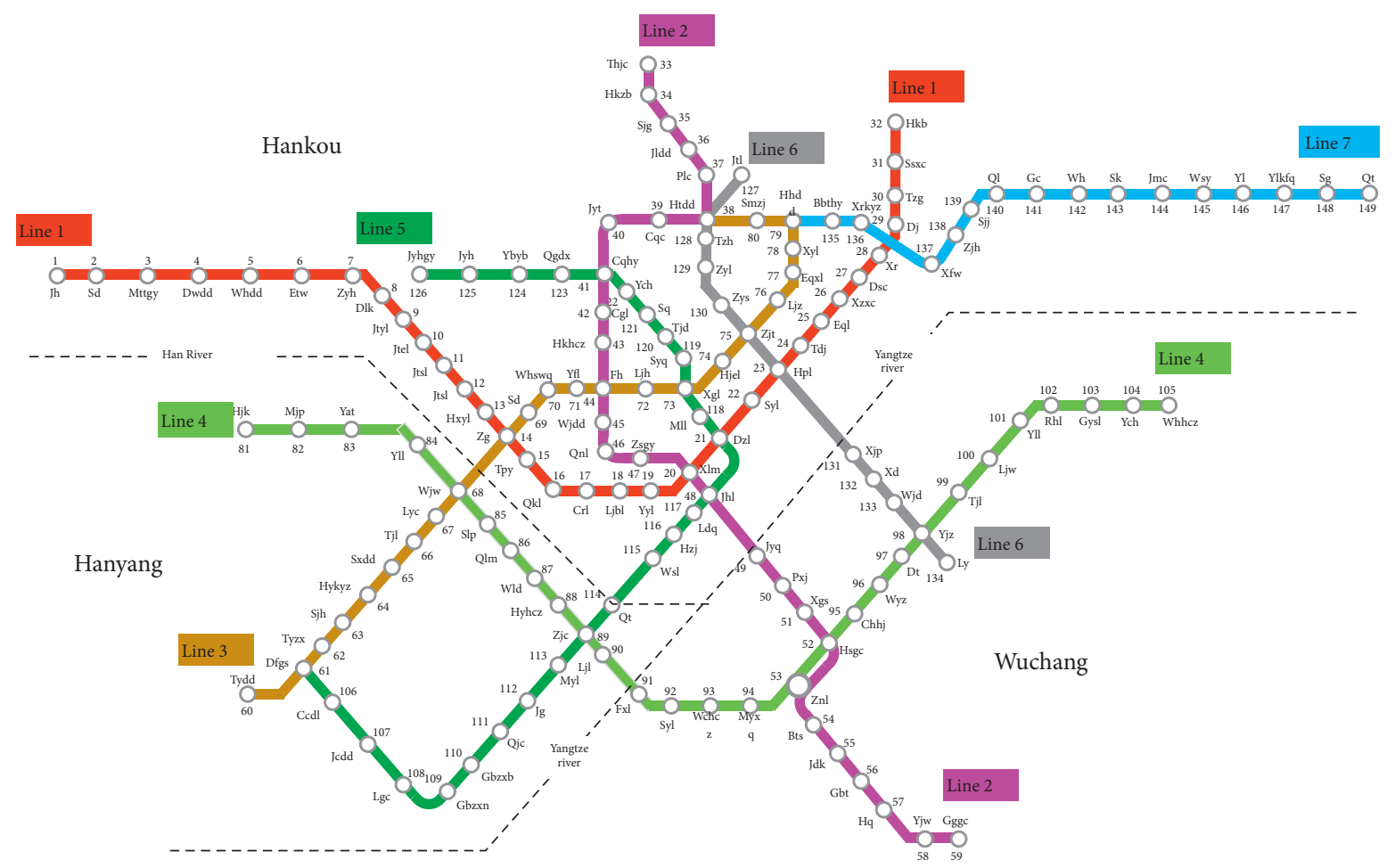

FIgURE 1: Wuhan's URT network in September 2018.

TABle 1: Transport capacities and average headway of lines.

\begin{tabular}{|c|c|c|c|c|c|c|}
\hline \multirow{2}{*}{ Lines } & \multicolumn{3}{|c|}{ Downstream } & \multicolumn{3}{|c|}{ Upstream } \\
\hline & Headway (min) & Capacity (trips/h) & Seats (seats/h) & Headway (min) & Capacity (trips/h) & Seats (seats $/ \mathrm{h}$ ) \\
\hline 1 & 3.8 & 23360 & 5600 & 4.6 & 20440 & 5950 \\
\hline 2 & 3.8 & 23360 & 5600 & 3.3 & 26280 & 6300 \\
\hline 3 & 4.3 & 20440 & 4900 & 3.8 & 23360 & 5600 \\
\hline 4 & 3.8 & 23360 & 5600 & 3.3 & 26280 & 6300 \\
\hline 5 & 3.3 & 26280 & 6300 & 3.8 & 23360 & 5600 \\
\hline 6 & 4.3 & 20440 & 4900 & 4.3 & 20440 & 5950 \\
\hline 7 & 6.0 & 14600 & 3500 & 7.5 & 11680 & 2800 \\
\hline
\end{tabular}

TABLE 2: Transfer walking time at transfer stations on line 1.

\begin{tabular}{lcccc}
\hline Station & $\begin{array}{c}\text { Transfer } \\
\text { direction }\end{array}$ & $\begin{array}{c}\text { Time } \\
\text { (min) }\end{array}$ & $\begin{array}{c}\text { Transfer } \\
\text { direction }\end{array}$ & $\begin{array}{c}\text { Time } \\
\text { (min) }\end{array}$ \\
\hline 14 & Line 1 to line 3 & 2.9 & Line 3 to line 1 & 2.6 \\
20 & Line 1 to line 2 & 2.5 & Line 2 to line 1 & 2.0 \\
21 & Line 1 to line 5 & 2.7 & Line 5 to line 1 & 2.7 \\
23 & Line 1 to line 7 & 2.9 & Line 7 to line 1 & 2.7 \\
\hline
\end{tabular}

returned, including 1302 valid questionnaires. The effective sampling rate of the questionnaires is $91.24 \%$, which meets the statistical requirements. The survey sample includes 697 men and 605 women. The surveyed passengers aged under 30 years old, between 30 and 60 years old, as well as over 60 years old account for $46.93 \%, 44.39 \%$, and $8.68 \%$, respectively, of total surveyed passengers. In addition, students (31.57\%), officials (9.68\%), businessmen (43.24\%), and workers $(15.51 \%)$ are surveyed. From the survey analysis, it is
TABLE 3: In-vehicle time of some links on line 1.

\begin{tabular}{cccccc}
\hline \multirow{2}{*}{ Link } & \multicolumn{2}{c}{ In-vehicle time (min) } & Link & \multicolumn{2}{c}{ In-vehicle time (min) } \\
& Downstream & Upstream & & Downstream & Upstream \\
\hline $1-2$ & 1.9 & 1.7 & $6-7$ & 1.6 & 1.2 \\
$2-3$ & 3.4 & 3.0 & $7-8$ & 1.4 & 1.6 \\
$3-4$ & 1.8 & 1.6 & $8-9$ & 2.5 & 2.5 \\
$4-5$ & 2.8 & 2.8 & $9-10$ & 2.6 & 3.1 \\
$5-6$ & 2.8 & 3.1 & $10-11$ & 1.6 & 1.4 \\
\hline
\end{tabular}

found that, during disturbances in Wuhan's URT network, passengers think their trips are acceptable only when their average relative increase in GTC is below 0.34 . Therefore, the preset threshold to determine passengers' categories is 0.34 . To compute passengers' GTC, the time weights, value of time, and fare are determined. The recommended default value for personal travel time should be $30 \%$ of household income per hour according to the economist Kenneth Gwilliam [40]. Therefore, the value of time $\alpha$ is estimated to 
be $16.54 ¥ /$ hour for passengers based on the household income of $161,000 ¥ /$ per year in Wuhan. The time weights are $\beta^{\text {wait }}=1.75, \beta^{\text {walk }}=1.75$, and $\beta^{\text {trans }}=8.35$ according to Cats and Jenelius [27]. $\beta^{\text {crow }}$ is related to the seat availability (sitting or standing) and crowding in the trains, which is shown in Table 4 [35]. The load factor is the ratio of passenger flow on a link to the number of seats on that link. It can indicate the crowding in the trains. The fare between an OD pair in Wuhan's URT is determined based on the shortest distances between that OD pair. The relation of fares to the shortest distance between an OD pair is shown in Table 5.

3.3. Network Performance in Normal Operation. A logitbased stochastic user equilibrium model is applied to assign passenger OD trips to Wuhan's URT network during evening peak hours. The passenger volume on links is shown in Figure 2, which shows that the links with high passenger volume are located at the center of Wuhan. In addition, the links which connect three parts of Wuhan (Wuchang, Hankou, and Hanyang) have high passenger volumes.

The passengers' actual travel time and perceived travel time on Wuhan's URT network during evening peak hours are computed after passenger trip assignment. The average travel time per trip and average perceived travel time per trip on Wuhan's URT network are 32.57 minutes and 68.92 minutes, respectively, during evening peak hours. Figure 3 shows that the perceived in-vehicle time is 1.93 times of actual in-vehicle time per trip. In addition, the increased perceived travel time is 7.73 minutes per trip due to transfer, which cannot be measured fully with actual travel time. Therefore, the perceived travel time can reflect more passengers' travel information and travel quality than actual travel time.

The average GTC per trip is $23.43 ¥$ which equals the fare (4.42 $¥$ ) plus the monetary value of perceived travel time (19.01 ¥). Passengers' total GTC is $8,156,325.79 ¥$ on Wuhan's URT network during evening peak hours according to 348,115 total passenger trips during evening peak hours. The components of the average GTC per trip are shown in Figure 4. It shows that the largest component of the GTC is the perceived in-vehicle time (53.97\%). The monetary values of perceived walking time, waiting time, and increased perceived time due to transfer are, respectively, $10.48 \%, 7.58 \%$, and $9.10 \%$ of the GTC on Wuhan's URT network during evening peak hours. The passengers' total GTC is computed with perceived travel time conveys service quality perceived by passengers (e.g., crowding in the vehicle, seat availability, perceived time components, and increased perceived travel time due to transfer).

\subsection{Every Line's Most Critical Link and Its Simulated Capacity} Reductions. The RAPT and betweenness of links are computed after the passenger OD trips assignment. Every line's most critical link on Wuhan's URT network during evening peak hours (shown in Table 6) is identified based on RAPT and betweenness.
The implicit assumptions of using betweenness of links to identify critical links in URT networks are that passenger trips between OD pairs are the same (all OD pairs are equally important) and all passengers choose the shortest paths to travel between OD pairs. However, the passenger trips between OD pairs vary greatly in the URT, and thus the weights of OD pairs are not the same. Passengers choose travel paths between OD pairs according to the GTC of paths. Therefore, not all passengers actually travel through the shortest paths. Critical links identification with RAPT considers the passenger trips between OD pairs and passengers' travel behavior. Therefore, Table 6 shows that the critical links identified with the betweenness and RAPT are quite different.

To simulate the link capacity reductions $\left(E_{\text {redu }}, x\right)$ that often occur on a URT network, capacity reductions on each line's most critical link and multiple lines' most critical links are simulated, respectively, on Wuhan's URT network during evening peak hours. It is assumed that the links are independent of each other in a simulation [11, 41], since it is difficult to estimate the effect of link capacity reductions on other links' capacities.

The capacity reductions on each line's most critical link are simulated when its capacity is reduced by $x$ (for $x$ values of $10 \%, 20 \%, 30 \%, \ldots, 90 \%, 100 \%)$. The capacities of the most critical links are reduced by the same ratio $x$ when capacity reductions on multiple lines' most critical links are simulated. The capacity reductions on the most critical links of two to seven lines, identified with RAPT and betweenness, respectively, are simulated. In addition, the capacity reduction links are determined by their rankings on RAPT and betweenness values, respectively, which are shown in Table 6 . For example, if the capacity reductions on three lines' most critical links identified with RAPT are simulated, then the three lines are lines 5, 1, and 3. After the capacities of lines' most critical links are reduced, a logit-based stochastic user equilibrium model is used to compute the passengers' GTC using equations (3) and (4). Then, the reliability and unreliability metrics of Wuhan's URT network during evening peak hours are computed.

\subsection{Results}

3.5.1. Reliability of Wuhan's URT When Capacity Reductions Occur on the Lines' Most Critical Links

(1) Capacity Reductions on Each Line's Most Critical Link. The reliability metric of Wuhan's URT network (FAT) is computed during evening peak hours when the capacity of each line's most critical link is reduced and is shown in Figure 5. The results show that the capacity reduction on line 1 to 5's most critical link identified with RAPT decrease the FAT on Wuhan's URT network faster than for the most critical link identified with betweenness. This indicates that critical links can be identified more accurately with RAPT than with betweenness. The reason is that the most critical links identified with RAPT have high passenger volume and are located at Wuhan's center. If their capacities decrease, 
TABLE 4: The value of $\beta^{\text {crow }}$ in different load factors.

\begin{tabular}{lcc}
\hline Load factor $(\%)$ & Sitting & $\beta^{\text {crow }}$ \\
& Standing \\
\hline $0-75$ & 0.86 & - \\
$75-100$ & 0.95 & - \\
$100-125$ & 1.05 & 1.62 \\
$125-150$ & 1.16 & 1.79 \\
$150-175$ & 1.27 & 1.99 \\
$175-200$ & 1.40 & 2.20 \\
$>200$ & 1.55 & 2.44 \\
\hline
\end{tabular}

TABle 5: Fares corresponding to the shortest distance between an OD pair.

\begin{tabular}{lccccccccc}
\hline Distance $(\mathrm{km})$ & $(0,4]$ & $(4,8]$ & $(8,12]$ & $(12,18]$ & $(18,24]$ & $(24,32]$ & $(32,40]$ & $(40,50]$ & $>50$ \\
\hline Price $(¥)$ & 2 & 3 & 4 & 5 & 6 & 7 & 8 & 9 & 10 \\
\hline
\end{tabular}

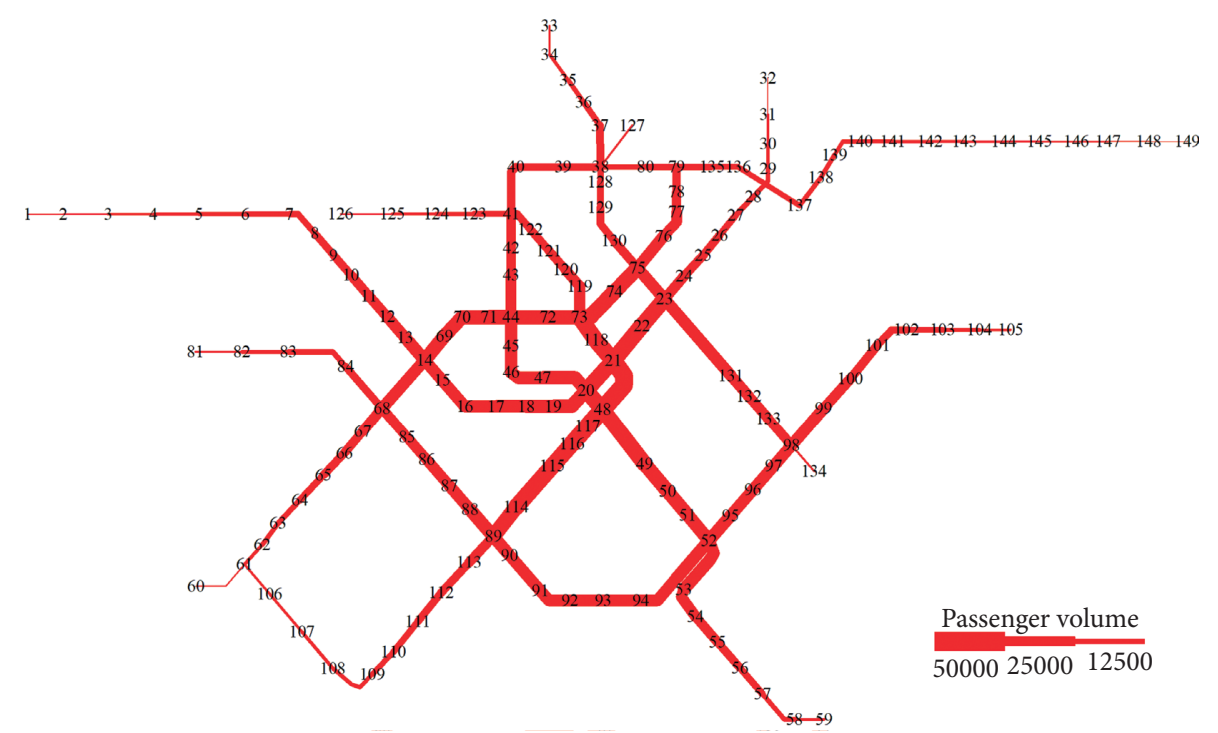

FIGURE 2: Passenger volume on Wuhan's URT links during evening peak hours.

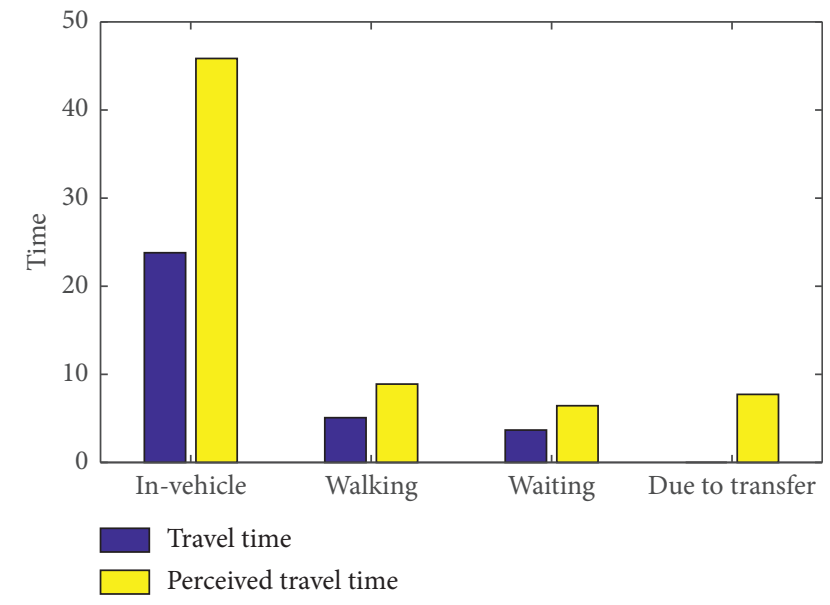

FIGURE 3: The components of the average travel time per trip and average perceived travel time per trip.

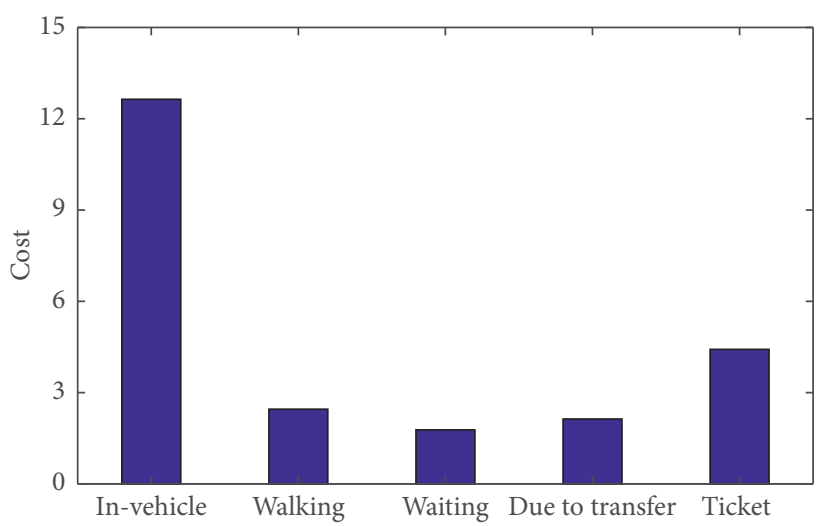

FIgURE 4: The components of the average GTC per trip. 
TABLE 6: Every line's most critical link on Wuhan's URT network during evening peak hours.

\begin{tabular}{|c|c|c|c|c|c|c|}
\hline \multirow{2}{*}{ Critical link } & \multicolumn{3}{|c|}{ Identified with RAPT } & \multicolumn{3}{|c|}{ Identified with betweenness } \\
\hline & Link (station-station) & RAPT (\%) & Rank & Link (station-station) & Betweenness (\%) & Rank \\
\hline On line 1 & $21-22$ & 12.60 & 2 & 13-14 & 9.06 & 5 \\
\hline On line 2 & $20-48$ & 12.32 & 4 & $53-54$ & 6.24 & 7 \\
\hline On line 3 & $73-72$ & 12.50 & 3 & $73-74$ & 16.38 & 1 \\
\hline On line 4 & $88-89$ & 9.83 & 5 & $98-99$ & 6.57 & 6 \\
\hline On line 5 & $48-117$ & 14.95 & 1 & $21-48$ & 12.04 & 3 \\
\hline On line 6 & $23-75$ & 8.98 & 6 & $132-133$ & 11.22 & 4 \\
\hline On line 7 & 79-135 & 4.79 & 7 & $135-136$ & 12.18 & 2 \\
\hline
\end{tabular}

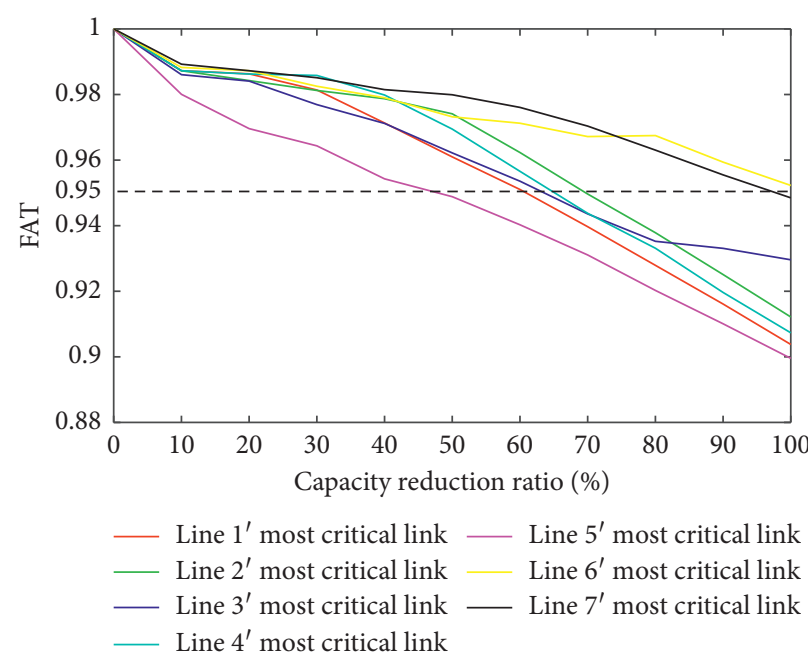

(a)

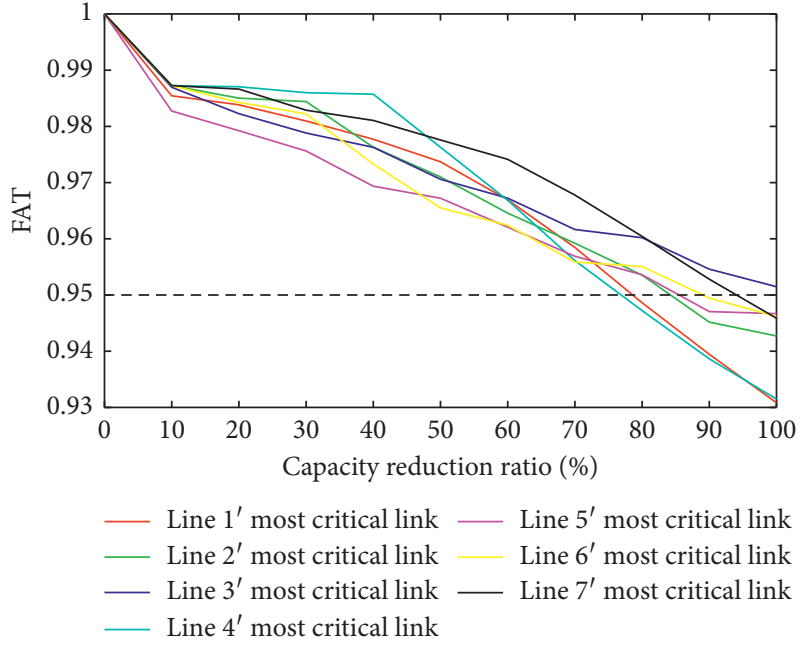

(b)

FIGURE 5: Reliability of Wuhan's URT network (a) when the capacity of each line's most critical link identified with RAPT is reduced and (b) when the capacity of each line's most critical link identified with betweenness is reduced.

then more passengers are shifted to categories II and III. Figure 5 illustrates that, as the capacity decreases, the FAT on Wuhan's URT network decreases slowly at first and then rapidly. The reason is that capacity reductions on a line's most critical link increase slowly the number of passengers who belong to category II when the capacity reduction ratio is below $50 \%$. However, if the capacity of a line's most critical link decreases by over $50 \%$, the passengers in category III increase rapidly due to their path flow exceeding path capacities.

The guidance for dealing with capacity reductions on each line's most critical link is obtained by analyzing Figure 5. For example, to maintain the high reliability of Wuhan's URT network during evening peak hours, i.e., guarantee that the FAT exceeds 0.95 , the operators should avoid capacity reductions below $50 \%$ in line 5 's most critical link which is identified with RAPT. Similarly, the capacity reduction ratio for another line's most critical link which corresponds to the networks' FAT of 0.95 can be determined. It can be used as a monitoring capacity reduction ratio to ensure the high reliability of Wuhan's URT network.

The effect of capacity reductions on a line's most critical link identified with RAPT on another line's reliability in Wuhan's URT network during evening peak hours is then analyzed. The FAT on a line is the fraction of passengers departing from that line and belonging to category I. The FAT on lines is computed when the capacity of a line's most critical link identified with RAPT is reduced by a ratio corresponding to the network's FAT value of 0.95 . The result, shown in Table 7, indicates that capacity reductions on a line's most critical link reduce the FAT not only on that line but also on other lines. Taking line 1's most critical link as an example, if its capacity is reduced by $60 \%$ (corresponding to the network's FAT value of 0.95), then the FAT on lines 1 and 5 decreases to 0.91 and 0.93 , respectively. The reason for the decreased reliability of line 5 is that passengers who ride line 5 from Hanyang to Hankou must pass through line 1's most critical link during evening peak hours. Thus capacity reductions on line 1's most critical link shift many passengers into categories II and III.

Some suggestions can be offered for mitigating the negative effect of capacity reductions on a line's most critical link on another line's reliability in Wuhan's URT network during evening peak hours. Taking line 1's most critical link as an example, if its capacity is reduced, then operators should emphasize prompt evacuation measures not only for line 1 but also for line 5 . It means operators should take measures to recover the capacity of line 1's most critical link 
TABLE 7: The FAT on lines when the capacity of a line's most critical link is reduced.

\begin{tabular}{lcccccccc}
\hline \multirow{2}{*}{ Critical link } & \multirow{2}{*}{ Capacity reduction ratio (\%) } & Line 1 & Line 2 & Line 3 & Line 4 & Line 5 & Line 6 & Line 7 \\
\hline Line 1's & & $\mathbf{0 . 9 1}$ & 0.96 & 0.99 & 0.99 & $\mathbf{0 . 9 3}$ & 0.97 & 0.97 \\
Line 2's & 60 & 0.97 & $\mathbf{0 . 9 2}$ & 0.99 & 0.99 & $\mathbf{0 . 9 5}$ & 0.99 & 0.98 \\
Line 3's & 60 & 0.99 & 0.98 & $\mathbf{0 . 8 7}$ & 0.98 & 0.96 & 0.98 & $\mathbf{0 . 9 2}$ \\
Line 4's & 60 & $\mathbf{0 . 9 6}$ & 0.98 & 0.97 & $\mathbf{0 . 9 2}$ & 0.95 & 0.99 & 0.98 \\
Line 5's & 40 & 0.96 & $\mathbf{0 . 9 5}$ & 0.98 & 0.99 & $\mathbf{0 . 7 9}$ & 0.97 & 0.97 \\
Line 6's & 90 & 0.99 & 0.98 & 0.96 & 0.98 & 0.96 & $\mathbf{0 . 7 0}$ & $\mathbf{0 . 9 3}$ \\
Line 7's & 90 & 0.99 & $\mathbf{0 . 9 5}$ & 0.96 & 0.97 & 0.97 & 0.98 & $\mathbf{0 . 7 1}$ \\
\hline
\end{tabular}

as soon as possible. Operators also need to transport passengers on line 5 rapidly by adding trains, enhancing passenger travel guidance and expediting security checks. Similarly, the priority line for which emergency measures should be taken can be determined from Table 7 when the capacity of a line's most critical link identified with RAPT is reduced.

(2) Capacity Reductions on Multiple Lines' Most Critical Links. The FAT on Wuhan's URT network during evening peak hours is computed when the capacities of multiple lines' most critical links are reduced, as shown in Figure 6. Figures 5 and 6 demonstrate that the capacity reductions on multiple lines' most critical links have a much stronger negative effect on the reliability of Wuhan's URT network than reductions on one line's most critical link. Figures 6(a) and 6(b) illustrate that the capacity reductions on multiple lines' most critical links identified with RAPT have a higher negative influence on the reliability of Wuhan's URT network than those caused by capacity reductions on multiple lines' most critical links identified with betweenness. The relation between network reliability, the number of a line's most critical links whose capacities are reduced, and the capacity reduction ratio shows that even if the number of a line's most critical links whose capacities are reduced increases to 7 , the FAT on Wuhan's URT network can be maintained at about 0.9 when the capacity reduction ratio is below $20 \%$. The reason for this result is that the ratio of passenger flow to capacities of lines' most critical links is nearly $80 \%$ on Wuhan's URT network during evening peak hours. If the capacities of lines' most critical links decrease by less than $20 \%$, then some passengers are shifted into category II and few passengers are shifted into category III. Therefore, the FAT is nearly 0.9 in such a case. However, if the capacity reduction ratio exceeds $20 \%$, many passengers should travel on new alternative paths because there is no spare capacity in their original paths. This shifts many passengers into category II and greatly decreases the FAT on Wuhan's URT network.

Some suggestions can be provided to operators by analyzing the result when the capacities of multiple lines' most critical links are reduced: to maintain the FAT on Wuhan's URT network above 0.9 , the capacities of lines' most critical links should not decrease by more than $20 \%$. The high reliability of Wuhan's URT network (whose FAT is over 0.95) can still be maintained if the capacities of the any two lines' most critical links are reduced by $20 \%$ or the capacities of any three lines' most critical links are reduced by $10 \%$.

\subsubsection{Unreliability of Wuhan's URT When the Critical Link Capacity Reductions Occur}

(1) Capacity Reductions on Each Line's Most Critical Link. The network's unreliability metric for Wuhan's URT network during evening peak hours is computed when the capacity of each line's most critical link is reduced, which is shown in Figure 7. The capacity reduction on line 1 to 5 's most critical link is identified with RAPT increase TGCR faster than for the most critical link identified with betweenness. Therefore, PAPT identifies lines' most critical links which greatly affect Wuhan's URT performance more accurately than betweenness. Figure 7 illustrates that capacity reduction on line 5's most critical link identified with RAPT significantly increases the total GTC and degrades the network performance.

To mitigate the negative impact of capacity reductions in a lines' most critical link on network performance of Wuhan's URT during evening peak hours, operators should prevent capacity reductions occurring on line 5's most critical link (link 48-117 identified with RAPT). For example, operators should strengthen the protection and inspection of lines' most critical links. To maintain the high performance of Wuhan's URT network, i.e., guarantee the TGCR below 0.05 , operators should avoid capacity reductions exceeding $10 \%$ on line 5 's most critical link. Similarly, the capacity reduction ratio for another line's most critical link which corresponds to network's TGCR of 0.05 can be determined. It can serve as a monitoring capacity reduction ratio to ensure the good performance of Wuhan's URT network.

The effect of capacity reductions on a line's most critical link identified with RAPT on another line's performance in Wuhan's URT network during evening peak hours is then analyzed. The impact on the line's performance is measured with the TGCR of passengers departing from that line. The TGCR on every line is computed when the capacity of a line's most critical link identified with RAPT is reduced by the capacity reduction ratio corresponding to network's TGCR of 0.05 . The result is shown in Table 8, which demonstrates that capacity reductions on a line's most critical link increase total GTC on that line and on another line. The capacity reduction ratio for line 7's most critical link corresponding to the network's TGCR of 0.05 is highest among the lines' most critical links. The reason is that line 7 is located in Wuhan's surrounding area which has low travel demand. The TGCR on line 6 is high when the capacity of line 7's most critical link is reduced by $60 \%$ (corresponding to network's 


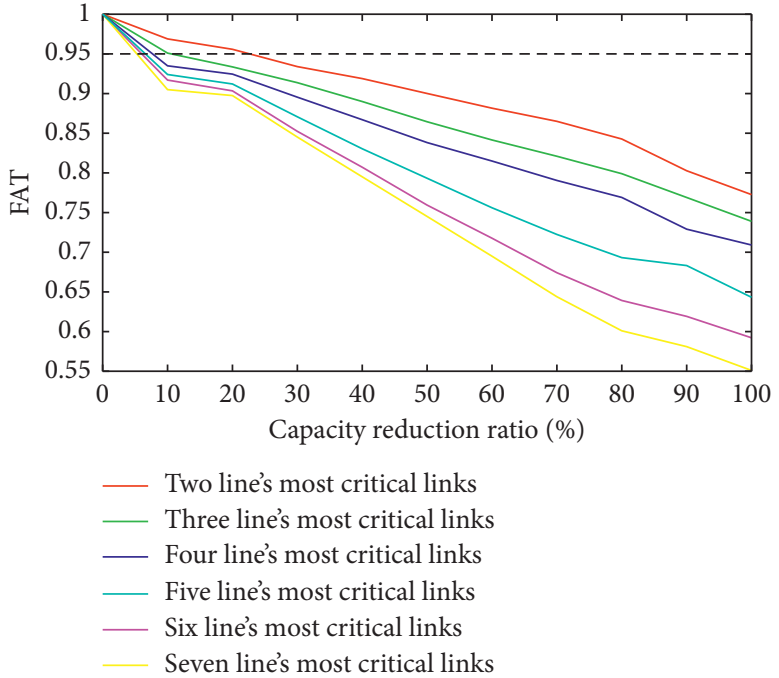

(a)

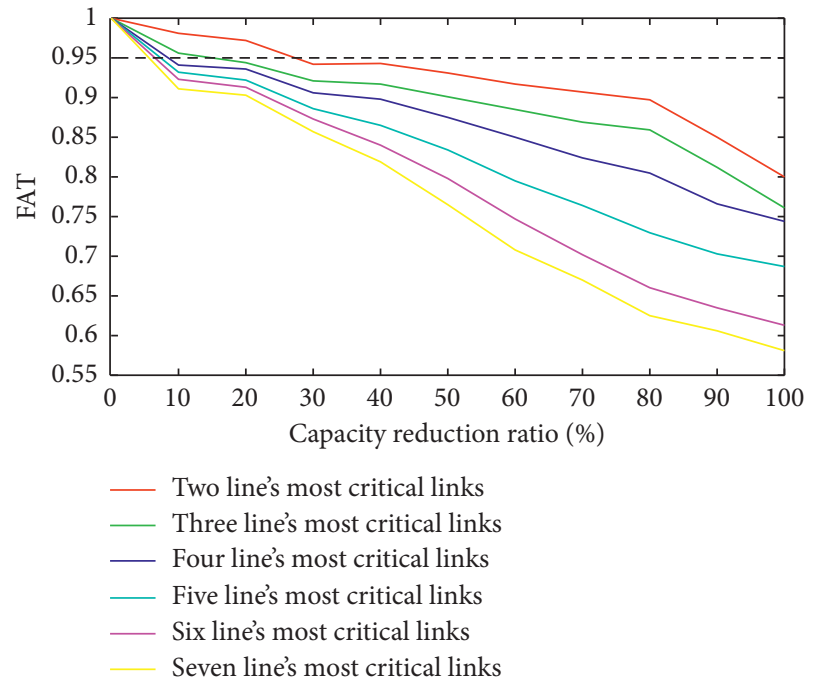

(b)

FIGURE 6: Reliability of Wuhan's URT network (a) when the capacities of multiple lines' most critical links identified with RAPT are reduced and (b) when the capacities of multiple lines' most critical links identified with betweenness are reduced.

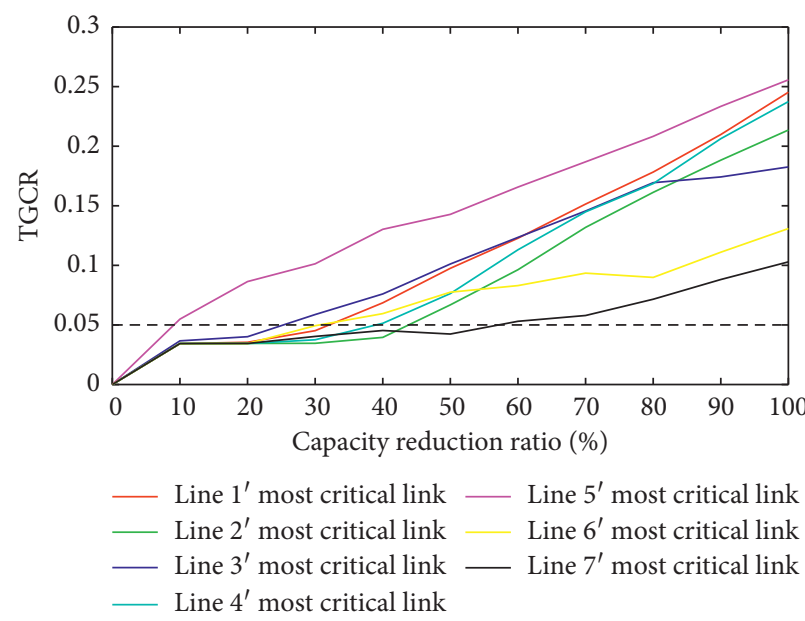

(a)

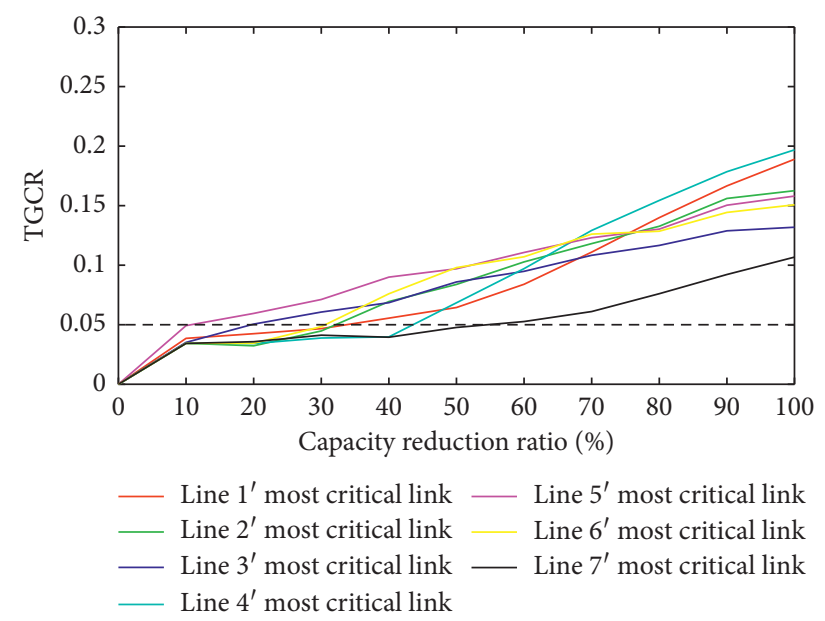

(b)

FIGURE 7: Unreliability of Wuhan's URT network (a) when the capacity of each line's most critical link identified with RAPT is reduced and (b) when the capacity of each line's most critical link identified with betweenness is reduced.

TGCR of 0.05), since it shifts many passengers into category III who depart from line 6 to line 7. Therefore, to maintain a high performance of Wuhan's URT network (with TGCR below 0.05 ), the capacity of line 7's most critical link should not decrease by over $60 \%$. If the capacity reductions occur on line 7's most critical link (link 79-135), then the emergency measures should be taken for lines 6 and 7 to avoid poor network performance. These measures include running buses between stations 79 and 135 to transport passengers who need travel through capacity reduction link; adding trains on line 6 , enhancing passenger travel guidance and expediting security checks on lines 6 and 7 to transport passengers on lines 6 and 7 as soon as possible. The priority line for which emergency measures should be taken can be determined from Table 8 when the capacity reductions occur on a line's most critical link.

(2) Capacity Reductions on Multiple Lines' Most Critical Links. The unreliability of Wuhan's URT network during evening peak hours is shown in Figure 8 when the capacities of multiple lines' most critical links are reduced. Figures 7 and 8 demonstrate that the capacity reductions on multiple lines' most critical links have a much stronger negative impact on the performance of Wuhan's URT network than that caused by capacity reductions on a line's most critical link. TGCR is high, and the network performance is poor when capacity reductions occur on multiple lines' most critical links. Figure 8 indicates that capacity reductions on 
TABLE 8: The TGCR on every line when the capacity of a line's most critical link is reduced.

\begin{tabular}{|c|c|c|c|c|c|c|c|c|}
\hline \multirow{2}{*}{ Critical link } & \multirow{2}{*}{ Capacity reduction ratio $(\%)$} & \multicolumn{7}{|c|}{ TGCR on every line } \\
\hline & & Line 1 & Line 2 & Line 3 & Line 4 & Line 5 & Line 6 & Line 7 \\
\hline Line 1 & 30 & 0.07 & 0.03 & 0.02 & 0.02 & 0.04 & 0.02 & 0.02 \\
\hline Line 2 & 40 & 0.02 & 0.05 & 0.01 & 0.02 & 0.03 & 0.01 & 0.01 \\
\hline Line 3 & 30 & 0.01 & 0.01 & 0.05 & 0.02 & 0.03 & 0.06 & 0.05 \\
\hline Line 4 & 30 & 0.02 & 0.04 & 0.01 & 0.06 & 0.02 & 0.02 & 0.01 \\
\hline Line 5 & 10 & 0.01 & 0.04 & 0.01 & 0.02 & 0.05 & 0.02 & 0.01 \\
\hline Line 6 & 40 & 0.01 & 0.01 & 0.01 & 0.01 & 0.02 & 0.11 & 0.06 \\
\hline Line 7 & 60 & 0.01 & 0.01 & 0.03 & 0.01 & 0.01 & 0.07 & 0.12 \\
\hline
\end{tabular}

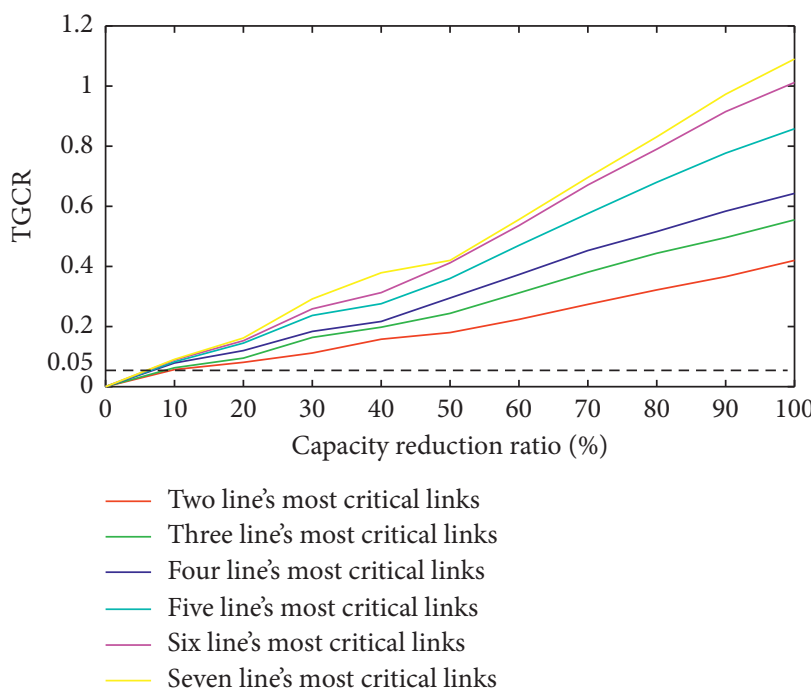

(a)

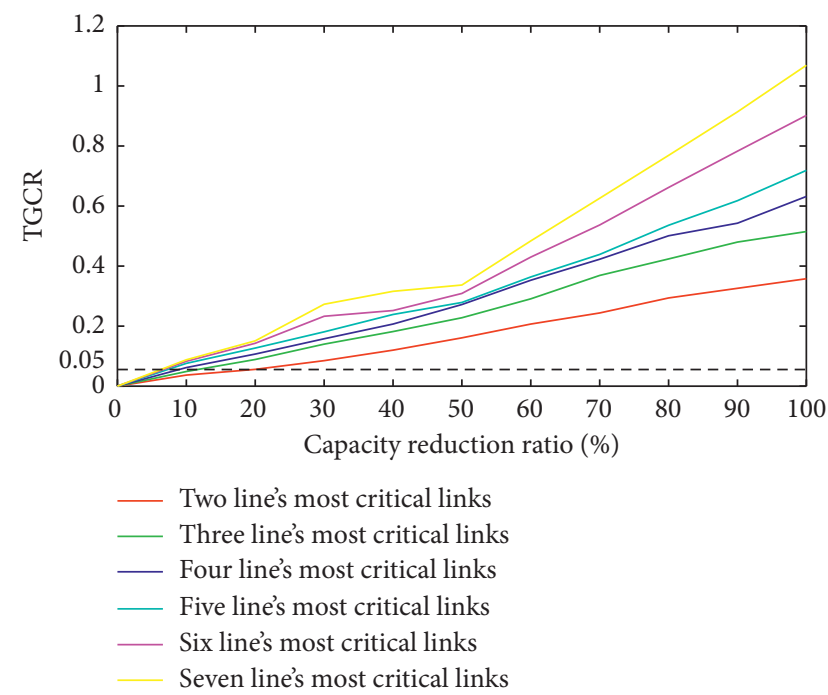

(b)

FIGURE 8: Unreliability of Wuhan's URT network (a) when the capacities of multiple lines' most critical link identified with RAPT are reduced and (b) when the capacities of multiple lines' most critical link identified with betweenness are reduced.

multiple lines' most critical links identified with RAPT have a higher negative influence on the performance of Wuhan's URT network than those identified with betweenness. The relation between network unreliability and capacity reduction ratio shows two inflection points when the capacity reduction ratio is $20 \%$ and $40 \%$. Two reasons can account for this result. One reason is that the ratio of passenger flow to capacities of lines' most critical links is nearly $80 \%$ on Wuhan's URT network during evening peak hours. The TGCR is low because the number of passengers in categories II and III is low when the capacities of multiple lines' most critical links decrease by less than $20 \%$. The other reason is that alternative paths exist on Wuhan's URT network when the capacity reduction ratio is between $20 \%$ and $40 \%$. Most passengers can travel on alternative paths when the capacities of multiple lines' most critical links decrease between $20 \%$ and $40 \%$. If the capacity reduction ratio exceeds $40 \%$, then the TGCR increases rapidly since many passengers are shifted into category III due to the passenger flows on alternative paths exceeding capacities.

Two important suggestions for operators may be stated based on the above analysis: to avoid total GTC increases exceeding $15 \%$ on Wuhan's URT network during evening peak hours, the capacity of multiple lines' most critical links should not decrease by more than $20 \%$. To avoid the high increase in total GTC and poor network performance of Wuhan's URT, operators should avoid having capacities of multiple lines' most critical links decrease by over $40 \%$.

\section{Conclusions}

This paper proposed measures for evaluating the reliability and unreliability of a URT network when the link capacity reductions occur. The most critical link on a line is identified with the proposed RAPT indicator. The passengers' GTC and total GTC are used to reflect service perceived by passengers and network performance, respectively. To measure the reliability of a URT network when link capacity reductions occur, the FAT and the TGCR are defined as the reliability and unreliability metrics, respectively. A logitbased stochastic user equilibrium model is applied to compute passengers' GTC and determine the passengers' categories.

Fully and partially reduced link capacities are both considered in analyzing the effect of capacity reductions in 
the lines' most critical links on the reliability of Wuhan's URT network during evening peak hours. The following conclusions may be drawn:

(1) The proposed RAPT indicator is effective in identifying lines' most critical links that greatly affect a URT's network reliability and performance.

(2) The capacity reduction ratio for each line's most critical link corresponding to networks' FAT of 0.95 and TGCR of 0.05 is determined. It can be used as a monitoring capacity reduction ratio to ensure high reliability and good performance of Wuhan's URT network.

(3) The priority line for which emergency measures should be taken can be determined after simulating capacity reductions on each line's most critical link. It can mitigate the negative effect of the capacity reductions in a line's most critical link on the reliability and performance of another line.

(4) In order to maintain the network reliability and network performance of Wuhan's URT above a certain level, the number of most critical links whose capacities are reduced in each line and the capacity reduction ratio that the network can withstand are determined. This can help operators in preparing emergency plans and assessing the harm caused by capacity reductions on lines' most critical links.

The reliability analysis of a URT network when link capacity reductions occur can facilitate the integration of measures for improving network reliability into URT network planning, operations, and real-time management. The identification of lines' most critical links also can provide guidance for infrastructure investment decisions and daily protection. Future studies may consider the probability of link capacity reductions and capacity recovery time. It is also desirable to further analyze how the impact of link capacity reductions on the reliability of a URT network can be mitigated.

\section{Abbreviations}

(1) Sets

S:

E:

$E_{\text {redu }}$ :

$P_{1}^{\mathrm{ij}}\left(E_{\text {redu }}, x\right)$,

$P_{2}^{\mathrm{ij}}\left(E_{\text {redu }}, x\right)$, and

$P_{3}^{\mathrm{ij}}\left(E_{\text {redu }}, x\right)$

$C_{\mathrm{ij}}$ :

The set of URT stations

The set of URT links

The capacity reduction link set

The sets of passengers from

station $i$ to station $j$ who belong to categories I, II, and III, respectively, when capacities of link set $E_{\text {redu }}$ are reduced by $x$ GTC set corresponding to paths from station $i$ to station $j$

\section{(2) Elements}

$i$ and $j$ : Stations belong to $S$

$e: \quad$ Link belongs to $L$

$C_{\mathrm{ij}}^{m}$ : The GTC corresponding to the path $m$ from station $i$ to station $j, C_{\mathrm{ij}}^{m} \in C_{\mathrm{ij}}$
(3) Parameters

$\kappa$ :

$\alpha:$

$\beta^{\text {wait }}, \beta^{\text {walk }}, \beta^{\text {crow }}$ and $\beta^{\text {trans }}$

$\theta$

$\delta_{e, m}^{\mathrm{ij}}:$

(4) Variables

$B_{e}$ :

$C(0,0)$ :

$C\left(E_{\text {redu }}, x\right):$

$C_{\mathrm{ij}}^{n}(0,0)$ :

$C_{\mathrm{ij}}^{n}\left(E_{\mathrm{redu}}, x\right):$

$C_{e}(\omega):$

$C_{\mathrm{ij}}^{m}$ :

$\mathrm{Cap}_{e}$ :

$c_{\mathrm{ij}}$ :

$E\left(C_{\mathrm{ij}}, \theta\right)$ :

$f_{e}:$

$\mathrm{fl}_{e}$ :

$I_{e}$ :

$n^{\text {trans }}(0,0)$ :

$n_{c}$ :

$n_{s}:$

$n_{\mathrm{ij}}$ :

$n_{\mathrm{ij}}^{e}:$

$\left|P_{1}^{\mathrm{ij}}\left(E_{\text {redu }}, x\right)\right|:$

$\left|p a_{\mathrm{ij}}\right|:$

$R_{\text {re }}\left(E_{\text {redu }}, x\right)$ :
The preset threshold

The value of time figure that converts perceived journey time into money

Values of time weights for waiting time, walking time, in-vehicle time, and transfer times, respectively

A nonnegative parameter represents the accuracy of passengers' perception of travel cost

A 0-1 binary variable; if the path $m$ from station $i$ to station $j$ contains the link $e$, then $\delta_{e, m}^{\mathrm{ij}}=1$; otherwise, $\delta_{e, m}^{\mathrm{ij}}=0$

Betweenness of link $e$ Passengers' total GTC when the URT network operates normally

Passengers' total GTC when capacities of link set $E_{\text {redu }}$ are reduced by $x$

Passenger $p_{\mathrm{ij}}^{n}$ 's GTC from station $i$ to station $j$ when the URT network operates normally

Passenger $p_{\mathrm{ij}}^{n}$ 's GTC from station $i$ to station $j$ when capacities of link set $E_{\text {redu }}$ are reduced by $x$

The functional relation between travel cost on a link and the link's passenger flow $\omega$

The GTC of the path $m$ from station $i$ to station $j$

The capacity of the link $e$

Fare from station $i$ to station $j$ The mathematical expectation of passengers for GTC perception The number of trains that traverse link $e$ in a given time period The passenger flow on link $e$ The ratio of affected passenger volume who is affected by link $e$ A passenger's transfer times when the URT system operates normally The number of passengers that a train can carry

The number of seats per train The number of shortest paths from station $i$ to station $j$

The number of shortest paths from station $i$ to station $j$ which contains link $e$

The number of passengers trips who travel from station $i$ to station $j$ and belong to category 1

The number of paths with spare capacity from station $i$ to station $j$ Metric of reliability of a URT system when capacities of link set $E_{\text {redu }}$ are reduced by $x$ 


\begin{tabular}{|c|c|}
\hline$R_{\text {un }}\left(E_{\text {redu }}, x\right):$ & $\begin{array}{l}\text { Metric of unreliability of a URT } \\
\text { system when capacities of link set } \\
E_{\text {redu }} \text { are reduced by } x\end{array}$ \\
\hline $\begin{array}{l}t^{\text {wait }}(0,0), t^{\text {walk }}(0,0) \text {, } \\
\text { and } t^{\text {inv }}(0,0) \text { : }\end{array}$ & $\begin{array}{l}\text { Waiting time, walking time, and in- } \\
\text { vehicle time, respectively, when the } \\
\text { URT system operates normally }\end{array}$ \\
\hline$t_{\mathrm{ij}}^{n}(0,0):$ & $\begin{array}{l}\text { Passenger } p_{\mathrm{ij}}^{n} \text { 's perceived travel time } \\
\text { from station } i \text { to station } j \text { when a } \\
\text { URT network operates normally }\end{array}$ \\
\hline$t_{e}^{\text {inv }}:$ & The in-vehicle time on link $e$ \\
\hline$v_{\mathrm{ij}}:$ & $\begin{array}{l}\text { The volume of passenger trip from } \\
\text { station } i \text { to station } j\end{array}$ \\
\hline$v_{\mathrm{ij}}^{m}:$ & $\begin{array}{l}\text { The volume of passenger trips from } \\
\text { station } i \text { to station } j \text { on the path } m\end{array}$ \\
\hline$x:$ & $\begin{array}{l}\text { Link capacity reduction ratio (the } \\
\text { unit is \%) }\end{array}$ \\
\hline$\psi_{e}:$ & $\begin{array}{l}\text { The number of seats on link } e \text { in a } \\
\text { given time period }\end{array}$ \\
\hline$\lambda:$ & $\begin{array}{l}\text { Relative increase in GTC for } \\
\text { passenger } p_{\mathrm{ij}}^{n} \text { when link capacity } \\
\text { reductions occur. }\end{array}$ \\
\hline
\end{tabular}

\section{Data Availability}

The data used to support the findings of this study are supplied by Yong Yin under license and so cannot be made freely available. The data can be made available from the corresponding author upon request (Yong Yin, yinyong@ swjtu.edu.cn).

\section{Conflicts of Interest}

The authors declare that there are no conflicts of interest regarding the publication of this paper.

\section{Acknowledgments}

This work was supported by the National Key R\&D Program of China (2017YFB1200700). The first author was supported by the China Scholarship Council (201907000071).

\section{References}

[1] T. Litman, "Impacts of rail transit on the performance of a transportation system," Transportation Research Record, vol. 1930, no. 1, pp. 21-29, 2005.

[2] G. Beirão and J. A. Sarsfield Cabral, "Understanding attitudes towards public transport and private car: a qualitative study," Transport Policy, vol. 14, no. 6, pp. 478-489, 2007.

[3] M. Cantwell, B. Caulfield, and M. O'Mahony, "Examining the factors that impact public transport commuting satisfaction," Journal of Public Transportation, vol. 12, no. 2, pp. 1-21, 2009.

[4] Y. Gu, X. Fu, Z. Liu, X. Xu, and A. Chen, "Performance of transportation network under perturbations: reliability, vulnerability, and resilience," Transportation Research Part E: Logistics and Transportation Review, vol. 133, Article ID 101809, 2020.

[5] M. A. P. Taylor, Vulnerability Analysis for Transportation Networks, Elsvier, Oxford, UK, 2017.

[6] L.-G. Mattsson and E. Jenelius, "Vulnerability and resilience of transport systems-a discussion of recent research,"
Transportation Research Part A: Policy and Practice, vol. 81, pp. 16-34, 2015.

[7] M. G. H. Bell, "A game theory approach to measuring the performance reliability of transport networks," Transportation Research Part B: Methodological, vol. 34, no. 6, pp. 533-545, 2000.

[8] H. Kim, C. Kim, and Y. Chun, "Network reliability and resilience of rapid transit systems," The Professional Geographer, vol. 68, no. 1, pp. 1-13, 2016.

[9] A. Chen, P. Kasikitwiwat, and C. Yang, "Alternate capacity reliability measures for transportation networks," Journal of Advanced Transportation, vol. 47, no. 1, pp. 79-104, 2013.

[10] H. Kim., Y. Song, and Y. Song, "Examining accessibility and reliability in the evolution of subway systems," Journal of Public Transportation, vol. 18, no. 3, pp. 89-106, 2015.

[11] Y. Yang, Y. Liu, M. Zhou, F. Li, and C. Sun, "Robustness assessment of urban rail transit based on complex network theory: a case study of the Beijing Subway," Safety Science, vol. 79, pp. 149-162, 2015.

[12] C. Jiang, L. Wu, F. Xu, and J. Yuan, "Characteristics and reliability analysis of the complex network in Guangzhou rail transit," Intelligent Automation \& Soft Computing, vol. 19, no. 2, pp. 217-225, 2013.

[13] Z. Q. Wang and R. H. Xu, "Reliability simulation analysis of urban rail transit networks based on complex network," Journal of System Simulation, vol. 21, no. 20, pp. 6670-6674, 2009.

[14] X. Zhang, E. Miller-Hooks, and K. Denny, "Assessing the role of network topology in transportation network resilience," Journal of Transport Geography, vol. 46, pp. 35-45, 2015.

[15] J. Liu, H. Lu, H. Ma, and W. Liu, "Network vulnerability analysis of rail transit plans in Beijng-Tianjin-Hebei region considering connectivity reliability," Sustainability, vol. 9, no. 8, pp. 2-17, 2017.

[16] Z. Zou, Y. Xiao, and J. Gao, "Robustness analysis of urban transit network based on complex networks theory," Kybernetes, vol. 42, no. 3, pp. 383-399, 2013.

[17] Z.-P. Du and A. Nicholson, "Degradable transportation systems: sensitivity and reliability analysis," Transportation Research Part B: Methodological, vol. 31, no. 3, pp. 225-237, 1997.

[18] D. M. Scott, D. C. Novak, L. Aultman-Hall, and F. Guo, "Network robustness index: a new method for identifying critical links and evaluating the performance of transportation networks," Journal of Transport Geography, vol. 14, no. 3, pp. 215-227, 2006.

[19] J. Liu, Q. Peng, J. Chen, and Y. Yin, “Connectivity reliability on an urban rail transit network from the perspective of passenger travel," Urban Rail Transit, vol. 6, no. 1, pp. 1-14, 2020.

[20] D. Sun., Y. Zhao, and Q.-C. Lu, "Vulnerability analysis of urban rail transit networks: a case study of Shanghai, China," Sustainability, vol. 7, no. 6, pp. 6919-6936, 2015.

[21] H. Kim. and Y. Song, "An integrated measure of accessibility and reliability of mass transit systems," Transportation, vol. 45, no. 4, pp. 1075-1100, 2018.

[22] Q.-C. Lu, "Modeling network resilience of rail transit under operational incidents," Transportation Research Part A: Policy and Practice, vol. 117, pp. 227-237, 2018.

[23] A. De-Los-Santos, G. Laporte, J. A. Mesa, and F. Perea, "Evaluating passenger robustness in a rail transit network," Transportation Research Part C: Emerging Technologies, vol. 20, no. 1, pp. 34-46, 2012. 
[24] J. Liu, P. M. Schonfeld, Q. Peng, and Y. Yin, "Measures of travel reliability on an urban rail transit network," Journal of Transportation Engineering, Part A: Systems, vol. 146, no. 6, 2020.

[25] M. Snelder, H. J. van Zuylen, and L. H. Immers, “A framework for robustness analysis of road networks for short term variations in supply," Transportation Research Part A: Policy and Practice, vol. 46, no. 5, pp. 828-842, 2012.

[26] Y. Jiang, Y. Wang, W. Y. Szeto, A. H. F. Chow, and A. Nagurney, "Probabilistic assessment of transport network vulnerability with equilibrium flows," International Journal of Sustainable Transportation, 2020.

[27] O. Cats and E. Jenelius, "Beyond a complete failure: the impact of partial capacity degradation on public transport network vulnerability," Transportmetrica B: Transport Dynamics, vol. 6, no. 2, pp. 77-96, 2016.

[28] E. Jenelius, "Public transport experienced service reliability: integrating travel time and travel conditions," Transportation Research Part A: Policy and Practice, vol. 117, pp. 275-291, 2018.

[29] N. van Oort, "Incorporating service reliability in public transport design and performance requirements: international survey results and recommendations," Research in Transportation Economics, vol. 48, pp. 92-100, 2014.

[30] E. I. Diab, M. G. Badami, and A. M. El-Geneidy, "Bus transit service reliability and improvement strategies: integrating the perspectives of passengers and transit agencies in north America," Transport Reviews, vol. 35, no. 3, pp. 292-328, 2015.

[31] N. A. Bruzelius, "Microeconomic theory and generalised cost," Transportation, vol. 10, no. 3, pp. 233-245, 1981.

[32] F. J. Cesario, "Value of time in recreation benefit studies," Land Economics, vol. 52, no. 1, 1976.

[33] L. Lesley, "Generalised costs and the value of time as a method of patronage forecasting," Acta Technica Jaurinensis, vol. 2, no. 1, pp. 57-68, 2009.

[34] L. Todd, "Valuing transit service quality improvements," Journal of Public Transportation, vol. 11, no. 2, pp. 43-63, 2008.

[35] M. Wardman and G. Whelan, “Twenty years of rail crowding valuation studies: evidence and lessons from British experience," Transport Reviews, vol. 31, no. 3, pp. 379-398, 2011.

[36] A. Chen, S. Pravinvongvuth, X. Xu, S. Ryu, and P. Chootinan, "Examining the scaling effect and overlapping problem in logit-based stochastic user equilibrium models," Transportation Research Part A: Policy and Practice, vol. 46, no. 8, pp. 1343-1358, 2012.

[37] H. Kato, Y. Kaneko, and M. Inoue, "Comparative analysis of transit assignment: evidence from urban railway system in the Tokyo metropolitan area," Transportation, vol. 37, no. 5, pp. 775-799, 2010.

[38] H. X. Liu, X. He, and B. He, "Method of successive weighted averages (MSWA) and self-regulated averaging schemes for solving stochastic user equilibrium problem," Networks and Spatial Economics, vol. 9, no. 4, pp. 485-503, 2009.

[39] M. Dixit, T. Brands, N. Van Oort, O. Cats, and S. Hoogendoorn, "Passenger travel time reliability for multimodal public transport journeys transportation research record," Journal of the Transportation Research Board, vol. 2673, no. 2, 2019.

[40] T. Litman, Transportation Cost and Benefit Analysis, p. 31, Victoria Transport Policy Institute, Victoria, Canada, 2009.

[41] O. Cats and E. Jenelius, "Vulnerability analysis of public transport networks: a dynamic approach and case study for Stockholm," in Proceedings of the 5th International Symposium on Transportation Network Reliability (INSTR2012), pp. 18-19, Hong Kong, China, December 2012. 